\title{
Specialized microbial databases for inductive exploration of microbial genome sequences
}

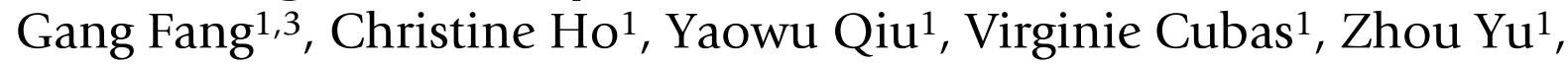 \\ Cédric Cabau1 ${ }^{1}$, Frankie Cheung1, Ivan Moszer ${ }^{2,3}$ and Antoine Danchin*1,3
}

\begin{abstract}
Address: ${ }^{1}$ HKU-Pasteur Research Centre, Dexter HC Man Building, 8, Sassoon Road, Pokfulam, Hong Kong, China, ${ }^{2}$ Plate-forme Intégration et Analyse Génomiques, Genopole, Institut Pasteur, 28 rue du Docteur Roux, 75724 Paris Cedex 15, France and 32unité de Génétique des Génomes Bactériens, CNRS URA2171, Institut Pasteur, 28 rue du Docteur Roux, 75724 Paris Cedex 15, France

Email: Gang Fang - fangg@pasteur.fr; Christine Ho - chrisho@hkucc.hku.hk; Yaowu Qiu - sunnyschool@126.com; Virginie Cubas - vcubs@yahoo.fr; ZhouYu - zhou.yu@utoronto.ca; Cédric Cabau - cedric.cabau@diamant.jouy.inra.fr; Frankie Cheung - ftcheung@hkusua.hku.hk; Ivan Moszer - moszer@pasteur.fr; Antoine Danchin* - adanchin@pasteur.fr

* Corresponding author
\end{abstract}

Published: 07 February 2005

BMC Genomics 2005, 6:14 doi:10.1186/1471-2164-6-14

This article is available from: http://www.biomedcentral.com/147I-2/64/6/14

(C) 2005 Fang et al; licensee BioMed Central Ltd.

This is an Open Access article distributed under the terms of the Creative Commons Attribution License (http://creativecommons.org/licenses/by/2.0), which permits unrestricted use, distribution, and reproduction in any medium, provided the original work is properly cited.
Received: 01 December 2004

Accepted: 07 February 2005

\begin{abstract}
Background: The enormous amount of genome sequence data asks for user-oriented databases to manage sequences and annotations. Queries must include search tools permitting function identification through exploration of related objects.

Methods: The GenoList package for collecting and mining microbial genome databases has been rewritten using MySQL as the database management system. Functions that were not available in MySQL, such as nested subquery, have been implemented.

Results: Inductive reasoning in the study of genomes starts from "islands of knowledge", centered around genes with some known background. With this concept of "neighborhood" in mind, a modified version of the GenoList structure has been used for organizing sequence data from prokaryotic genomes of particular interest in China. GenoChore http://bioinfo.hku.hk/ genochore.html, a set of 17 specialized end-user-oriented microbial databases (including one instance of Microsporidia, Encephalitozoon cuniculi, a member of Eukarya) has been made publicly available. These databases allow the user to browse genome sequence and annotation data using standard queries. In addition they provide a weekly update of searches against the world-wide protein sequences data libraries, allowing one to monitor annotation updates on genes of interest. Finally, they allow users to search for patterns in DNA or protein sequences, taking into account a clustering of genes into formal operons, as well as providing extra facilities to query sequences using predefined sequence patterns.
\end{abstract}

Conclusion: This growing set of specialized microbial databases organize data created by the first Chinese bacterial genome programs (ThermaList, Thermoanaerobacter tencongensis, LeptoList, with two different genomes of Leptospira interrogans and SepiList, Staphylococcus epidermidis) associated to related organisms for comparison. 


\section{Background}

We are facing a deluge of genome sequences. As of January 14th, 2005, the GOLD site identified 1248 completed or ongoing genome programs http://www.genomeson line.org, and this certainly reflects only a partial view of the existing programs. While this shows that we implicitely possess an enormous wealth of information about the functions carried out by genes and genomes, the very fact that this amount is enormous makes it extremely difficult to mine that information easily. The role of specialized databases is to make this task easier for end-users. Many types of microbial genome databases exist. Most of them have been developed in a context of bioinformatics centres or laboratories purely favoring in silico research rather than the coupling between experiments using computers and experiments at the bench, and this is reflected in the structure and aims of the databases [1-8]. In contrast, at the onset of genome programs, we decided to set up a data structure for bacterial genomes that would help experimentalists to access knowledge on genes and genomes in an end user-oriented fashion. This was first the aim of the Colibri project, with the goal to organize Escherichia coli genome data, well before the whole genome sequence was known [9]. Later on, the SubtiList database was at the core of the Bacillus subtilis genome program data access [10]. Many databases constructed on the GenoList data schema were subsequently constructed (http://genolist.pasteur.fr, [11]). However, with the exponentially growing set of genome sequences, it became important to divide up the work while maintaining the main goal of the project, that of being end-user-driven and of course, user-friendly. While an ongoing effort aims at integrating all bacterial genomes within the GenoList frame into a single database, it is important to create individual databases that could be regularly updated by a selected team of scientists (preferably those that initiated the corresponding genome program). This is particularly important for countries that are beginning to develop at a high speed into the genomics era. We took the opportunity of the creation of the HKU-Pasteur Research Centre in Hong Kong (China) to set up genome databases for the microbial sequencing projects developed in China (with databases for related organisms for comparison). Within this economic context, it was also important to take into account the cost of development. The paradigm GenoList databases are based on commercial DataBase Management Systems (DBMS) [11] and we decided to shift from a commercial DBMS to a non-commercial one, providing more freedom for the future of the project. In the present set of databases (GenoChore), emphasis is placed on retrieval of information centered on the gene as the central object, with exploration methods that query simple properties of the gene products (such as molecular mass or isoelectric point) in addition to more complex features such as the class of codon usage bias used in the gene [12].
Furthermore, queries can be made on the sequence itself using large scale analyses such as BLAST, and search for word patterns present in DNA and protein sequences.

\section{Construction and content Data schema}

Because we wished to shift from a commercial DBMS to an open-source one, there were some applications that could not be implemented readily due to the lack of certain advantages possessed by the commercial DBMSs. Hence, we had to alter the data structure in order to cope with this situation. The core data schema used in this work was that of GenoList version 3.1 [11], with slight modifications (Figure 1).

\section{Database management system}

In the present GenoList databases, the DBMS used is Sybase $^{\mathrm{TM}}$. While this is convenient because of excellent stability and maintenance, this may pose problems in terms of commercial policies, especially if the structure has to be exported. We therefore decided to rewrite the management of the GenoList structure using MySQL http:// www.mysql.com/. Most function transfers were straightforward. However some functions such as nested subquery that were not available in MySQL had to be dealt with indirectly. The nested subquery has been entirely circumvented in the PERL code and is dealt with in the Extended Search algorithm by concatenating different SQL queries simply using the "AND" or "OR"connector.

\section{Data input}

Sequence and annotation data were parsed from the files extracted from the International Nucleotide Sequences Database (INSD: DDBJ/EMBL-EBI/GenBank [13,14]) with the following procedure. To get access to the INSD, the authors of a genome sequence must follow the specification of the Feature Table Definition (FTD) jointly issued by the INSD partners http:// www.ncbi.nlm.nih.gov/projects/collab/FT/index.html.

The current version is Version 6.2 Oct 15, 2004. While this specification is rigid, there is still a significant degree of freedom in annotation, so that a large number of individual situations have to be taken care of semi-automatically. The basic idea of the parser is firstly to read through the input file at the INSD and check file formats. Subsequently, the information is collected and distributed into several temporary files using a set of predefined keywords and their qualifiers (i.e. those characterizing the data schema). Subsequently, a check process is initiated to identify all situations that do not fit the specifications, so that they can be corrected manually. Usually, most of the process of creating tables is automatic and only a few exceptions have to be corrected individually. A second type of input is also provided as an interactive interface to tell the database curator what information has to be 


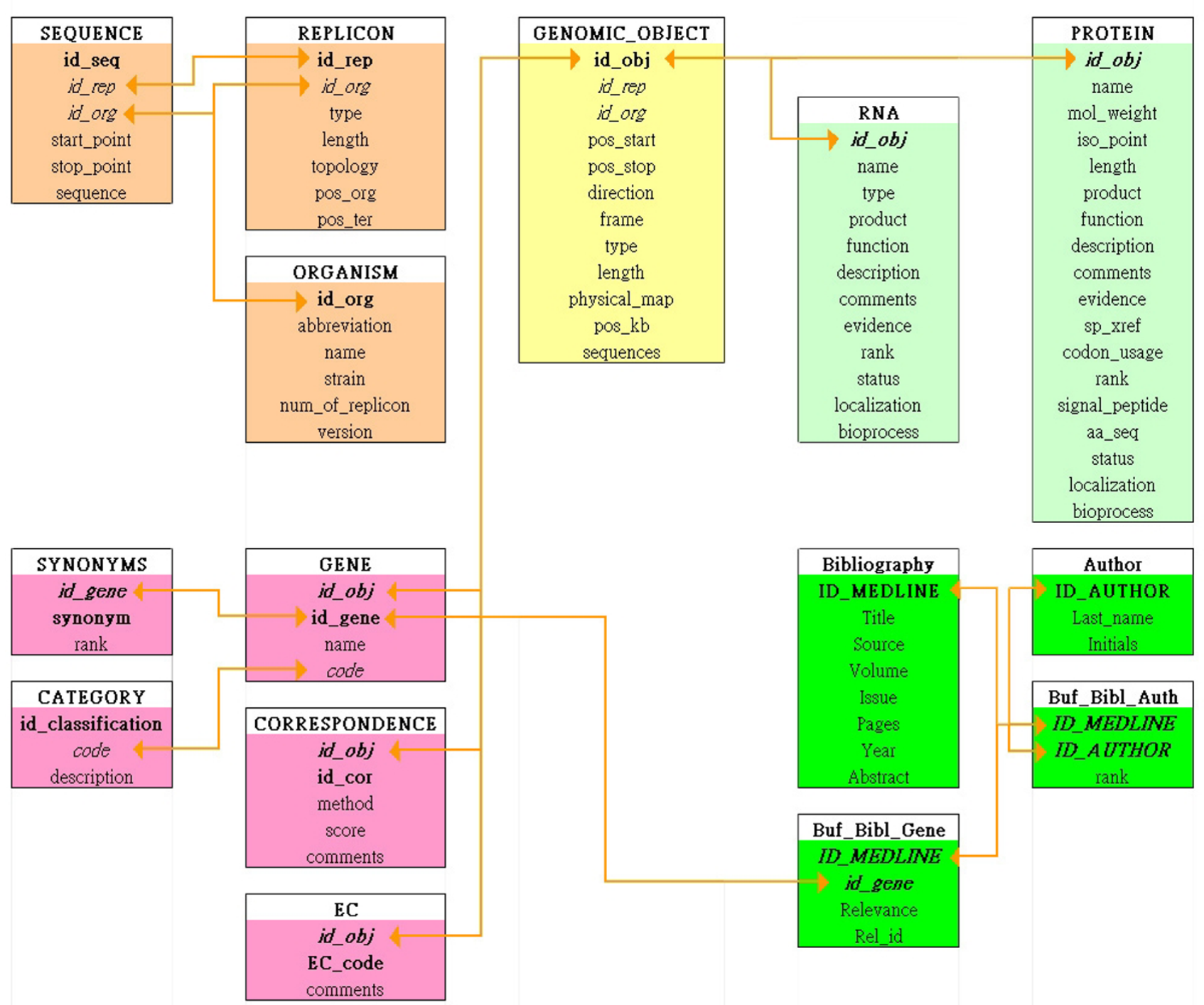

Figure I

Data Schema of the Databases. The core object of the schema is the Genomic_object, as in GenoList. It uses pointers in the sequence that delimits several categories of objects, including protein Coding DNA Sequences (CDSs), RNAs and other objects such as transcription terminators or riboswitches.

collected: once collected this information can be loaded into the databases directly (Figure 2). Teraprot data are obtained from Infobiogen http://www.infobiogen.fr/serv ices/Teraprot/.

\section{Query methods and interface}

We kept the interface of GenoList as published, except that a box providing access to protected curation of annotations is now provided, aiming initially at helping the first party (sequencing teams) annotators. The front page is made of three frames. Briefly, the vertical frame on the left contains the controls necessary to get access to the content of the database. The upper part of this frame contains text fields for querying the database according to five types of queries: gene name(s), chromosome region around a gene, chromosome region defined by positions, free text, functional classification (more detailed information about each type of query can be obtained by clicking on the question mark near the query title). The "Extended Search" button gives access to a search form allowing the user to perform multicriteria searches on all the database fields. The lower part of this frame allows one to launch 


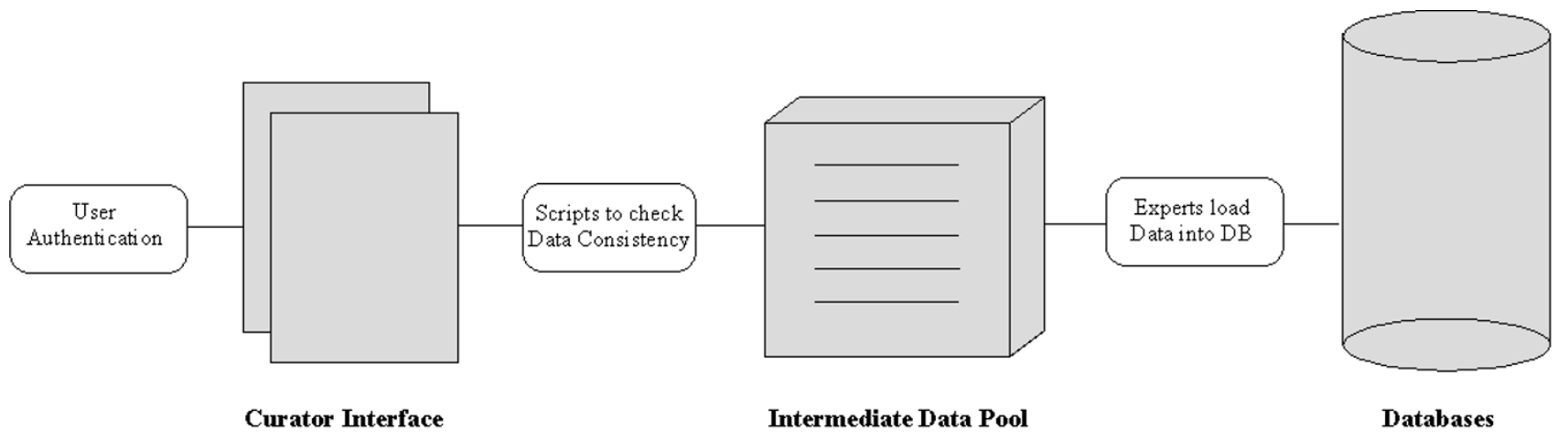

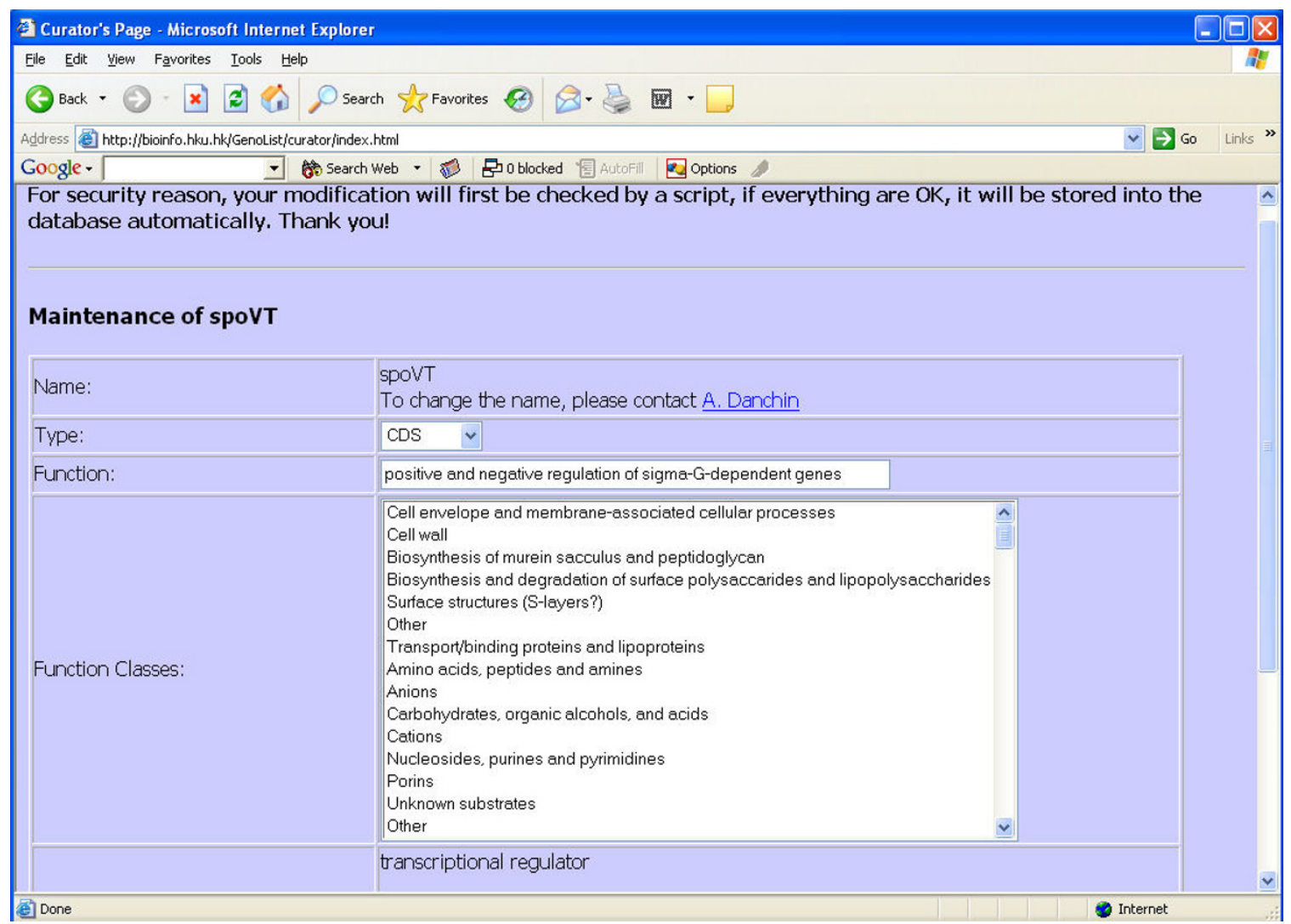

Figure 2

Implementation of a Database Curator Page. In order to help users who would participate in the improvement of the database annotation a Curator Page is provided permitting input of updated information. It is available to users after acceptation of their collaboration, through a password protected access. Once data consistency has been verified the new annotations are implemented in the current database.

sequence analysis tools: BLAST and FASTA database searches (on the sequence data), and DNA or protein pattern searches. In the former case, the user can choose to explore sequences located upstream of putative operons. In the latter case, the user can search for patterns anywhere in proteins, but also restrict the search to the beginning or 
end of the protein. The upper frame on the right can contain various types of information, depending on the genome and on the query. It can contain a graphical representation of a chromosome region, that can be obtained in several ways: usually from a gene in the bottom frame. This frame may contain launch forms and result lists from the sequence analysis tools available (pattern search, BLAST or FASTA scanning). The bottom frame on the right always contains detailed information about one given gene, including regularly updated BLAST searches and Teraprot reports as well as related bibliographic references. The original package managing the interface of GenoList databases was written in $\mathrm{C} / \mathrm{C}++$, following the first database schema [10], that had been adapted for use with the Sybase $^{\text {TM }}$ DBMS (the initial platform was using the DBMS $4^{\text {th }}$ Dimension $\left.^{\mathrm{TM}}\right)$. The modification of the database schema needed for using MySQL required additional adaptations of the application interfaces. Using the original package would have required iterative work that was systematically adding complexity into the system. Current best Web interfaces and application interfaces (i.e. friendly for sharing parties) are often based on Perl scripts. For this reason a new core management script was recreated, written in Perl, while keeping the package architecture and the Web interfaces. Among other languages that have comparable functionalities, the choice of Perl to create the system was motivated by its powerful capability to glue different programs or scripts together. In addition it is widely used by the INSD, and at the European Bioinformatics Institute in particular within the BioSapiens program http://www.biosapiens.info/. Furthermore, this choice allowed us to keep the optimized fast $\mathrm{C}$ code that has been constructed for searching pattern (strings of symbols) inside the DNA or amino acids sequences. The GenoList $\mathrm{C} / \mathrm{C}++$ package chose to use the GD library http://www.boutell.com/gd/ for generating graphic representations of genome regions. The GD graphics library is an open source library which allows programmers to easily generate PNG, JPEG, and WBMP images from many different programming languages. We used here a newer version of the same library (perl module perl-GD version 2.11) to make use of its improvements in creating dynamic pictures. In rewriting the core of the program we used the Perl module DBI http://dbi.perl.org/about/. A DBI is a middle layer between the outside applications and the communicator (DBD). Different DBMSs have their own communication mechanism to talk with outside applications, and in the present version the choice of the DBI module has been implemented in such a way that we could change the DBMS if necessary with minimal work. In this way, when changing the DBMS, it will only be necessary to tell the DBI about the specifications of the new DBMS without having to modify any other code. Finally, we used the Perl module CGI to facilitate the production of the WebPage interfaces. As a consequence fur- ther developments of the GenoChore package should be performed with minimal effort.

\section{Utility and discussion Data schema}

In the original GenoList structure, the central table corresponding to genomic objects carried all relevant features that are associated to genes and gene products. For the sake of future developments and to accommodate new feature annotation present in genome flatfiles, we separated this table into several gene product tables, specific for RNAs and proteins. The current data structure remains open to include tables for other types of data, such as regulation properties annotations when they will become available. Figure 1 displays a diagram of the current generic database schema (we did not show tables that remain empty for want of annotation data). As expected for a database meant to provide knowledge from genome programs, the central tables are focussing on genomic objects, the main one corresponding to protein Coding DNA Sequences (CDSs). To match this structure, the information present in the flat files created by the sequencing consortia, and present in the INSD, is split into three parts, namely, a) genomic objects, i.e. what we see in a chromosome, at precisely identified positions in the genome sequence (depending on the annotation tools available to the consortia), such as a CDS, a promoter, a terminator, a tRNA, an sRNA etc.; b) genome annotations, i.e. protein, RNA and other bio-molecules' products, functions, comments and so on; c) relations between genomic objects: e.g. the typical concept of gene requires its association to a promoter, a terminator and usually a CDS. In this representation, a set of genome objects' identifiers (ids) is used to represent a gene. This facilitates the association of genomic objects together with much more sophisticated relationships into more complex structures, when required.

It is important here to notice that, in contrast to a rather ubiquitous practice, we explicitely separate between Open Reading Frames (ORFs) that are simply sequences multiple of 3 between two termination codons (TAA, TAG and TGA) and CDSs, that begin with a specific codon, usually ATG (in the DNA text), preceded by a ribosome binding site (RBS), typically AAGGAGGT in many bacterial genomes. One must remember that in most genomes the beginning of CDSs has not been experimentally identified. Identification of CDS starts is however much easier in low $\mathrm{G}+\mathrm{C}$ Firmicutes that do not possess a counterpart of ribosomal protein $\mathrm{S} 1$ found in gamma proteobacteria [15]. In the same way, G+C-rich organisms have usually long ORFs, but the CDSs they harbour are usually highly enriched in $\mathrm{A}+\mathrm{T}$ at the third codon position. Some caution, therefore, should be exerted by users when using the information collected in the databases about the 
beginning of proteins in these organisms (for example in the Streptomyces coelicolor database, CoeliList).

\section{Nomenclature: naming genes}

Users know that the system used for naming genes in genome databases is extremely unwieldy and completely lacks standardization. This is usually because genes are simply labelled in databases by access numbers corresponding to the annotation phase of the relevant genome program (e.g. PA3004 for a gene found in the genome of Pseudomonas aeruginosa). In the absence of knowledge of a gene name it takes some time to identify it (often using BlastP), for example when aiming at the study of its neighborhood ((i.e. proximity of an object or a relationship with others sharing the same conceptual space, including presence in a common article [12]). Naturally, because most genes have never been experimentally identified in the majority of the newly sequenced genomes, this approach is certainly safer than giving a name without proper identification criteria. However it is extremely useful for scientists studying a genome to start from "islands of knowledge", with genes with a known background, reflected by a known gene (and a gene name has usually been coined by experimentalists for that gene). For this reason, we decided to use a conservative approach, using bidirectional best Blast hits of the genome of interest with model genome (Escherichia coli $\mathrm{K} 12$ and Bacillus subtilis 168). Orthologues were identified as reciprocal best hits [16] (using a global alignment where the gaps on the edges of the largest sequence are ignored) with at least $50 \%$ identity in amino acid sequence and less than $20 \%$ difference in protein length. When possible, in order to increase the likelihood of the putative identification we used a second well known representative of the genome under study and looked for orthologues between every pair of each of the two triplets (i.e. between each pair of the three organisms: the organism of which the database is constructed, B. subtilis for Firmicutes and another organism of the same family, such as Listeria monocytogenes, and E. coli for gamma-proteobacteria, with another one of the same family, such as Photorhabdus luminescens). Finding putative orthologues in the three organisms was considered as substantiating evidence for the use of a gene name. Then, in each triplet, we did not transfer the model organism gene name to all orthologues that were not simultaneously present in the three genomes or that gave different correspondences in different comparisons. In another comparison where the orthologues were found with at least $50 \%$ similarity, the model organism gene names to be transferred were preceded by the letter ' $y$ '. In order to help users recognize gene names (and all the knowledge they associate with those names) we used as reference names those in the model bacteria, trying to comply as much as possible with the names used at Swiss-
Prot in the HAMAP project http://www.expasy.org/sprot/ hamap/slides/hamap.html[17].

This allows the users to have "anchor" points to start to use the databases in a more efficient way. Naturally, the names previously used in the corresponding genome programs are kept as synonyms, so that access to the sequences with these names is still allowed. For example, in AeruList, gene rpsA can be accessed directly or using its synonym PA3162: it is then found downstream of $\mathrm{cmk}$ (a context similar to that found in many Gram negative bacteria) and upstream of himD. We are aware that some erroneous identification (or propagation of erroneous identifications) must have occurred in some cases, but we think that this is a trade-off (which will be continuously corrected) for a much more user-friendly usage of the databases. A ' $y$ ' letter starting a gene name indicates that it has not been experimentally identified, nor convincingly identified after in silico analysis yet. We provide curation pages (see below) to help users to correct annotation errors and improve annotation in a continuous way.

\section{Functional categories and bioprocesses}

An important feature for allowing users to explore biological functions is to investigate the genes neighborhoods [12]. Related functions are often coded by genes in close vicinity in the chromosome. We therefore used the GenoList table for functional categories, that allows the user to make links with the roles of proteins in the cell. The functional classification used in some of the present databases has been created by superimposing the functional classification (ontology) created for SubtiList, and that of Escherichia coli created by Monica Riley and her collaborators [18] (Additional file). In addition we created a field for the ontology describing underlying bioprocesses: explore, sense, shape, circulate, excrete, replicate, grow, respire, manage energy, store, scavenge, maintain, protect, control. They will be used in the future to color the arrows indicating genes in the picture of the region surrounding a gene of interest, allowing the user, at a glance, to have a rough idea of the processes encoded in the corresponding region.

\section{Queries using mining algorithms}

In addition to using keyword queries or sequence tags (such as molecular mass or isoelectric point of a protein) the database provides a versatile way to identify sequences from the biological knowledge viewpoint. In particular, as in many other databases, it allows the user to use Fasta, BlastP and BlastN to compare a sequence of interest to that of those present in the database. Furthermore, in contrast to most cases, it allows the user to extract information using motifs, that can be either continuous or discontinuous (e.g. finding all proteins with motif $\left.\mathrm{CXXCHX}_{12-25} \mathrm{C}\right)$. This facility has already, in a quite 
unobtrusive but efficient way, permitted discovery of many unexpected functions. We have also provided means to explore the beginning and the end of protein sequences, as well as DNA regions upstream of putative operons, computed as strings of genes transcribed in the same orientation and separated by a maximum number of nucleotides ( $60 \mathrm{nt}$ by default).

\section{Automatic updates}

Genome annotation is continuously updated by scientists all over the world, at a time when new genome sequences appear every three days or so. In order to cope with this enormous flux of information, a facility for browsing automatically new entries in major data libraries has been implemented. In the gene information panel, where each gene of interest is described after being identified as the result of a query (including resulting from a Blast or Pattern search), an "Automatic Blast" link provides a list of updated blast searches against the UniProt library (SWISSPROT+TREMBL). In addition, when the genome belongs to the 'Teraprot' Smith and Waterman Z-score family http://www.infobiogen.fr/services/Teraprot/, the corresponding links (that are statistically much more significant than the results of Blast searches) are provided, allowing the user to look for remote kinships.

To discuss the use of the databases we shall restrict our exploration to two databases from the package. LeptoList, that comprises two genomes (each one having two chromosomes) for Bacteria, and CunicuList, that describes the genome sequence and annotation of a small eukaryote.

\section{An example: LeptoList}

LeptoList is the reference database dedicated to the genome of Leptospira interrogans serovar Lai, the paradigm of leptospirosis causative agents [19]. It is presented together with a second sequence, that of $L$. interrogans serovar Copenhageni in order to allow easy comparison [20]. The WWW interface takes into account the fact that L. interrogans has two chromosomes (this feature was not yet displayed in GenoList databases). Using the regular comparison to the CDS to the non-redundant INSD protein database allowed us to suspect that a significant proportion of the short putative CDSs in the genome are likely ORFs and not authentic CDSs. This fits with the recent sequencing of the second Leptospira genome $[20,21]$.

A couple of examples of its use are given here. We looked for counterparts of RRF, the ribosome release factor. In order to find the gene we used a known sequence, from $B$. subtilis (frr gene product) and compared it using BLAST with the functionality implemented in LeptoList. This search led to a single gene, LA3295, located downstream of gene pyrH (as in most other bacterial genomes). This synteny is obviously highly significant. In the same way, the gene immediately upstream from pyrH (LA3297), as in other bacteria, is likely to be coding for elongation factor EFTs (tsf). When curating the database, we suggest to the curator that it would be of excellent policy to replace the gene numbers by the corresponding gene name. In another type of investigation, looking for patterns of the type TTGACA ( 1 ambiguity) - $17 \mathrm{nt}$ - TATAAT\# ( 1 ambiguity) (consensus sequence of the $\sigma^{70}$-type promoter) in the $300 \mathrm{nt}$ region upstream of genes revealed 70 sequences in chromosome I, many of which are likely to be promoters (at least they would be good guesses to start investigating promoters in L. interrogans). In the same way, the putative DNA binding site located in the $300 \mathrm{nt}$ nucleotide region upstream of genes, TGTGA ( 1 ambiguity) - $2 \mathrm{nt}-\mathrm{KK}-2 \mathrm{nt}$ - TCACA (1 ambiguity) (consensus sequence of the CAP/FNR family of transcriptional regulators), yielded 130 matches in chromosome I of serovar Lai and 72 matches in serovar Copenhageni and 2 in chromosome II of serovar Lai and 0 in serovar Copenhageni, allowing one to start investigating possible regulatory elements.

This result is interesting as it suggests that chromosome I genes are submitted to a regulation recognizing that particular DNA-protein binding site. Furthermore, most genes found with the site in serovar Copenhageni are also found in serovar Lai, with sometimes several repeats in the latter, occuring upstream of some genes (such as $\mathrm{fadH}$ or $\operatorname{prf}($ ), accounting for the higher total number of putative binding sites in that organism. It seems most interesting that genes involved in the control of respiration (cytochrome c biosynthesis), control of the TCA cycle (pyruvate dehydrogenase synthesis), control of the coupling between translation and transcription (stringent control) or translation itself (release factor 3 synthesis) are present in the list. While there are several putative adenylyl cyclase genes present in the organism, as well as several homologs of $\mathrm{crp}$, it is plausible to propose that cAMP plays an important role in the life cycle of $L$. interrogans, perhaps suggesting ways to allow multiplication on plates of this elusive organism.

LeptoList is accessible at the URL http://bioinfo.hku.hk/ GenoList/index.pl?database=leptolist

\section{CunicuList: a database for a small eukaryote genome}

The GenoList structure has been initially constructed for organizing sequence data from prokaryotic genomes. However it may be extended to other organisms as well (the "genomic object" type must be extended accordingly). We have therefore tested the implementation of the structure for the genome of Encephalitozoon cuniculi, belonging to the Microsporidia taxon. Eleven chromosomes are present in this organism. Extraction of 
Table I: List of databases present at the Bioinfo server The GenoChore suite presented here manage bacterial genome data, except for CunicuList, which presents the sequence and annotation data of the small eukaryote Encephalitozoon cuniculi.

\begin{tabular}{|c|c|c|}
\hline AeruList & Pseudomonas aeruginosa PAOI & $\begin{array}{l}\text { EMBL:AE00409I } \\
\text { http://www.ncbi.nlm.nih.gov/entrez/ } \\
\text { query.fcgi?db=genome\&cmd=search\&term=AE00409I }\end{array}$ \\
\hline AnthraList & Bacillus anthracis str. Ames & $\begin{array}{l}\text { EMBL:AEOI } 6879 \\
\text { http://www.ncbi.nlm.nih.gov/entrez/ } \\
\text { query.fcgi?db=genome\&cmd=search\&term=AE0I } 6879\end{array}$ \\
\hline CampyloList & Campylobacter jejuni NCTC III 68 & $\begin{array}{l}\text { EMBL:ALI I I I } 68 \\
\text { http://www.ncbi.nlm.nih.gov/entrez/ } \\
\text { query.fcgi?db=genome\&cmd=search\&term=AL I | | |68 }\end{array}$ \\
\hline CereList & Bacillus cereus ATCC 14579 & $\begin{array}{l}\text { EMBL:AEOI } 6877 \\
\text { http://www.ncbi.nlm.nih.gov/entrez/ } \\
\text { query.fcgi?db=genome\&cmd=search\&term=AE0I } 6877\end{array}$ \\
\hline CholeList & Vibrio cholerae & $\begin{array}{l}\text { EMBL:AE003852 } \\
\text { http://www.ncbi.nlm.nih.gov/entrez/ } \\
\text { query.fcgi?db=genome\&cmd=search\&term=AE003852, EMBL:AE003853 } \\
\text { http://www.ncbi.nlm.nih.gov/entrez/ } \\
\text { query.fcgi?db=genome\&cmd=search\&term=AE003853 }\end{array}$ \\
\hline CoeliList & Streptomyces coelicolor A3(2) & $\begin{array}{l}\text { EMBL:AL645882 } \\
\text { http://www.ncbi.nlm.nih.gov/entrez/ } \\
\text { query.fcgi?db=genome\&cmd=search\&term=AL645882 }\end{array}$ \\
\hline DiphteList & Corynebacterium diphtheriae NCTC 13129 & $\begin{array}{l}\text { EMBL:BX248353 } \\
\text { http://www.ncbi.nlm.nih.gov/entrez/ } \\
\text { query.fcgi?db=genome\&cmd=search\&term=BX248353 }\end{array}$ \\
\hline CunicuList & Encephalitozoon cuniculi & $\begin{array}{l}\text { EMBL:AL39|737 } \\
\text { http://www.ncbi.nlm.nih.gov/entrez/ } \\
\text { query.fcgi?db=genome\&cmd=search\&term=AL39|737, EMBL:AL590442 } \\
\text { http://www.ncbi.nlm.nih.gov/entrez/ } \\
\text { query.fcgi?db=genome\&cmd=search\&term=AL590442, EMBL:AL590443 } \\
\text { http://www.ncbi.nlm.nih.gov/entrez/ } \\
\text { query.fcgi?db=genome\&cmd=search\&term=AL590443, EMBL:AL590444 } \\
\text { http://www.ncbi.nlm.nih.gov/entrez/ } \\
\text { query.fcgi?db=genome\&cmd=search\&term=AL590444, EMBL:AL590445 } \\
\text { http://www.ncbi.nlm.nih.gov/entrez/ } \\
\text { query.fcgi?db=genome\&cmd=search\&term=AL590445, EMBL:AL590446 } \\
\text { http://www.ncbi.nlm.nih.gov/entrez/ } \\
\text { query.fcgi?db=genome\&cmd=search\&term=AL590446, EMBL:AL590447 } \\
\text { http://www.ncbi.nlm.nih.gov/entrez/ } \\
\text { query.fcgi?db=genome\&cmd=search\&term=AL590447, EMBL:AL590448 } \\
\text { http://www.ncbi.nlm.nih.gov/entrez/ } \\
\text { query.fcgi?db=genome\&cmd=search\&term=AL590448, EMBL:AL590449 } \\
\text { http://www.ncbi.nlm.nih.gov/entrez/ } \\
\text { query.fcgi?db=genome\&cmd=search\&term=AL590449, EMBL:AL590450 } \\
\text { http://www.ncbi.nlm.nih.gov/entrez/ } \\
\text { query.fcgi?db=genome\&cmd=search\&term=AL590450, EMBL:AL59045। } \\
\text { http://www.ncbi.nlm.nih.gov/entrez/ } \\
\text { query.fcgi?db=genome\&cmd=search\&term=AL59045। }\end{array}$ \\
\hline InfluList & Haemophilus influenzae Rd KW20 & $\begin{array}{l}\text { EMBL: } 442023 \\
\text { http://www.ncbi.nlm.nih.gov/entrez/ } \\
\text { query.fcgi?db=genome\&cmd=search\&term }=L 42023\end{array}$ \\
\hline LeptoList & Leptospira interrogans Lai str. $5660 \mathrm{I}$ & $\begin{array}{l}\text { EMBL:AE0 } 0300 \\
\text { http://www.ncbi.nlm.nih.gov/entrez/ } \\
\text { query.fcgi?db=genome\&cmd=search\&term=AEOI0300, EMBL:AEOI } 030 \text { I } \\
\text { http://www.ncbi.nlm.nih.gov/entrez/ } \\
\text { query.fcgi?db=genome\&cmd=search\&term=AE0I030I }\end{array}$ \\
\hline & Leptospira interrogans Fiocruz LI-130 & $\begin{array}{l}\text { EMBL:AE0 I } 6823 \\
\text { http://www.ncbi.nlm.nih.gov/entrez/ } \\
\text { query.fcgi?db=genome\&cmd=search\&term=AEO } \mid 6823, \text { EMBL:AEO I } 6824 \\
\text { http://www.ncbi.nlm.nih.gov/entrez/ } \\
\text { query.fcgi?db=genome\&cmd=search\&term=AE0 } 16824\end{array}$ \\
\hline MeningoList & Neisseria meningitidis MC58 & $\begin{array}{l}\text { EMBL:AE002098 } \\
\text { http://www.ncbi.nlm.nih.gov/entrez/ } \\
\text { query.fcgi?db=genome\&cmd=search\&term=AE002098 }\end{array}$ \\
\hline PutidaList & Pseudomonas putida KT2440 & $\begin{array}{l}\text { EMBL:AE0 I } 545 \text { I } \\
\text { http://www.ncbi.nlm.nih.gov/entrez/ } \\
\text { query.fcgi?db=genome\&cmd=search\&term=AE0 I } 545 \mid\end{array}$ \\
\hline
\end{tabular}


Table I: List of databases present at the Bioinfo server The GenoChore suite presented here manage bacterial genome data, except for CunicuList, which presents the sequence and annotation data of the small eukaryote Encephalitozoon cuniculi. (Continued)

\begin{tabular}{|c|c|c|}
\hline SepiList & Staphylococcus epidermidis ATCC 12228 & $\begin{array}{l}\text { EMBL:AE0I5929 } \\
\text { http://www.ncbi.nlm.nih.gov/entrez/ } \\
\text { query.fcgi?db=genome\&cmd=search\&term=AE0I5929 }\end{array}$ \\
\hline SubtiList & Bacillus subtilis str. 168 & $\begin{array}{l}\text { EMBL:AL009| } 26 \\
\text { http://www.ncbi.nlm.nih.gov/entrez/ } \\
\text { query.fcgi?db=genome\&cmd=search\&term=AL009|26 }\end{array}$ \\
\hline ThermaList & Thermoanaerobacter tencongensis MB4 & $\begin{array}{l}\text { EMBL:AE00869| } \\
\text { http://www.ncbi.nlm.nih.gov/entrez/ } \\
\text { query.fcgi?db=genome\&cmd=search\&term=AE00869| }\end{array}$ \\
\hline VulnifiList & Vibrio vulnificus YJ0I6 & $\begin{array}{l}\text { EMBL:BA000037 } \\
\text { http://www.ncbi.nlm.nih.gov/entrez/ } \\
\text { query.fcgi?db=genome\&cmd=search\&term=BA000037, EMBL:BA000038 } \\
\text { http://www.ncbi.nlm.nih.gov/entrez/ } \\
\text { query.fcgi?db=genome\&cmd=search\&term=BA000038 }\end{array}$ \\
\hline$\underline{\text { XylelList }}$ & Xylella fastidiosa $9 \mathrm{a} 5 \mathrm{c}$ & $\begin{array}{l}\text { EMBL:AE003849 } \\
\text { http://www.ncbi.nlm.nih.gov/entrez/ } \\
\text { query.fcgi?ddb=genome\&cmd=search\&term=AE003849 }\end{array}$ \\
\hline
\end{tabular}

information is similar to that from other databases. For example we looked for counterparts of genes involved in tRNA modification (often essential genes). Using MesJ (TilS) [22] as well as TrmU [23] we found that gene Ecu03_1240 is most probably involved in driving the codon and amino acid specificity of a tRNA (possibly isoleucine or lysine tRNA). In the same way we could predict that gene Ecu07_1610 codes for synthesis of dihydrouridine in tRNA, a general feature of tRNA structure, because of its similarity with the yacF B. subtilis gene. Looking for counterparts of genes in the methionine salvage pathway [24], we failed to identify any gene that would code for the enzymes of the pathway, indicating that the parasite obtains all the metabolites derived from S-adenosylmethionine from its host. This is substantiated by the fact that the genes needed to synthesize queuosine [25] are apparently absent from the genome. Some organisms do not use this major tRNA modification, but this could be an interesting information for identification of drug targets against the parasite, since this suggests that those metabolites have to be transported into the cell by specific permeases.

\section{Database curation}

Several other similar bacterial databases are accessible at URL http://bioinfo.hku.hk/genochore.html. Table 1 presents the list of microbial databases that are available at the Bioinfo server of the University of Hong Kong.

Despite of - or because of - the large number of genome programs, once a sequence has been deposited at the INSD, its annotation is seldom updated. This is because the cost of curating annotations is extremely high, and usually not considered, despite its enormous importance. One of our aims was therefore to allow curation by selected teams by creating a curator page where such teams would input their annotations, that would then be propagated to the databases. The basic schema of the curator interface is shown in Figure 2. In order to preserve the quality of the input data, potential users are asked to write to the database's webmaster to ask for account and passwords. We kept the interface of GenoList as published, except that a box providing access to protected curation of annotations is now provided, aiming initially at helping the first party (sequencing teams) annotators. If this works to our satisfaction this will be extended to selected third party annotators. Subsequently, on a yearly basis (or more frequently if needed) the collected re-annotation of the curators would be submitted as a new version of the same genome to the INSD. We hope that this service will be useful for the scientific community as a whole.

\section{Conclusions}

A set of 17 specialized end-user-oriented microbial databases (including one instance of Microsporidia) has been implemented in Hong Kong. They allow one to browse genome sequence and annotation data using the most frequent queries that end-users would like to ask. In addition they provide a weekly update of searches against the world-wide protein sequences data libraries, allowing one to monitor annotation on genes of interest. Finally, they allow users to search for patterns in DNA or protein sequences present in the databases. All comments, bug reports and suggestions for improvement are more than welcome: this work is meant to be useful for the community of microbiologists interested in genomics.

\section{Competing interests}

The author(s) declare that they have no competing interests. 


\section{Authors' contributions}

GF wrote the parsers used to create the preliminary $\mathrm{C} / \mathrm{C}++$ MySQL package, and created important sections of the Perl package; $\mathrm{CH}$ created the procedure for renaming orthologs with reference to accepted names for model bacteria (Bacillus subtilis and Escherichia coli), created the link to Teraprot for identification of gene functions, and implemented parts of the PERL package; YQ implemented parts of the Perl package; CC and VC implemented most of the databases into the core structure; ZY wrote part of the new parsers, implemented the two chromosomes of LeptoList by changing the data structure in the database and set up with CC the first LeptoList database; FC set up and administered the Apache web server and MySQL database; IM over the years designed most of the GenoList data schema and user interface; $\mathrm{AD}$ was at the origin of the project, participated in the design and evolution of the data schema, was the systematic tester and end-user and wrote the core of the article.

\section{Additional material}

\section{Additional File 1}

Functional categories. The genes' roles are listed into six major categories. The three first ones are directly linked to biological roles, while the remaining categories are created ad hoc: adaptation to atypical conditions correspond to miscellaneous roles, while the two last categories correspond to roles that have not yet been ascribed to genes because of lack of in vivo or in silico data

Click here for file

[http://www.biomedcentral.com/content/supplementary/14712164-6-14-S1.doc]

\section{Acknowledgements}

These databases are implemented at the Hong Kong University Computer Centre ( $\mathrm{Dr} \mathrm{Nam} \mathrm{Ng}$, Director). This effort is sponsored by the Innovation and Technology Fund of the government of the SAR Hong Kong, China (program BIOSUPPORT), granted to A. Danchin for the creation of the HKU-Pasteur Research Centre, with the collaboration of N. Ng, Computer Centre. We thank the CNRS (URA 2I7I, Genetics of Genomes) for support. GF was supported by the Friends of the Institut Pasteur in Hong Kong. Initial development stages of GenoList were performed in the framework of the European B. subtilis genome project (European Commission Biotechnology program - contracts BIO2-CT93-0272, BIO2-CT94-20II, BIO4CT96-0655), coordinated by F. Kunst and supported by the BACillus Industrial Platform (BACIP). We thank the contribution at various stages of $P$. Glaser, A. Hénaut, C. Médigue, M. Pupin, and A. Viari. We acknowledge the fruitful collaboration with Infobiogen and with A. Bairoch and the SWISSPROT team. In the most recent part of this work we benefited from the BioSapiens EU grant LSHG-CT-2003-503265.

\section{References}

I. Fumoto M, Miyazaki S, Sugawara H: Genome Information Broker (GIB): data retrieval and comparative analysis system for completed microbial genomes and more. Nucleic Acids Res 2002, 30:66-68.
2. Karp PD, Riley M, Saier M, Paulsen IT, Paley SM, Pellegrini-Toole A: The EcoCyc and MetaCyc databases. Nucleic Acids Res 2000, 28:56-59.

3. Meyer F, Goesmann A, McHardy AC, Bartels D, Bekel T, Clausen J, Kalinowski J, Linke B, Rupp O, Giegerich R, Puhler A: GenDB--an open source genome annotation system for prokaryote genomes. Nucleic Acids Res 2003, 3 I:2 187-2195.

4. Peterson JD, Umayam LA, Dickinson T, Hickey EK, White O: The Comprehensive Microbial Resource. Nucleic Acids Res 200I, 29:123-125.

5. Tateno Y, Imanishi T, Miyazaki S, Fukami-Kobayashi K, Saitou N, Sugawara H, Gojobori T: DNA Data Bank of Japan (DDBJ) for genome scale research in life science. Nucleic Acids Res 2002, 30:27-30.

6. Uchiyama I: MBGD: microbial genome database for comparative analysis. Nucleic Acids Res 2003, 31:58-62.

7. Wheeler DL, Church DM, Edgar R, Federhen S, Helmberg W, Madden TL, Pontius JU, Schuler GD, Schriml LM, Sequeira E, Suzek TO, Tatusova TA, Wagner L: Database resources of the National Center for Biotechnology Information: update. Nucleic Acids Res 2004, 32:D35-40.

8. Rudd KE: EcoGene: a genome sequence database for Escherichia coli K-12. Nucleic Acids Res 2000, 28:60-64.

9. Medigue C, Viari A, Henaut A, Danchin A: Colibri: a functional data base for the Escherichia coli genome. Microbiol Rev 1993, 57:623-654.

10. Moszer I, Glaser P, Danchin A: SubtiList: a relational database for the Bacillus subtilis genome. Microbiology 1995, I4I ( Pt 2):26I-268.

II. Moszer I, Jones LM, Moreira S, Fabry C, Danchin A: SubtiList: the reference database for the Bacillus subtilis genome. Nucleic Acids Res 2002, 30:62-65.

12. Nitschke P, Guerdoux-Jamet P, Chiapello H, Faroux G, Henaut C, Henaut A, Danchin A: Indigo: a World-Wide-Web review of genomes and gene functions. FEMS Microbiol Rev 1998, 22:207-227.

13. Miyazaki S, Sugawara H, Gojobori T, Tateno Y: DNA Data Bank of Japan (DDBJ) in XML. Nucleic Acids Res 2003, 3 I: I3-16.

14. Brunak S, Danchin A, Hattori M, Nakamura H, Shinozaki K, Matise T, Preuss D: Nucleotide sequence database policies. Science 2002, 298:1333

15. Danchin A: Comparison between the Escherichia coli and Bacillus subtilis genomes suggests that a major function of polynucleotide phosphorylase is to synthesize CDP. DNA Res 1997, 4:9-18.

16. Tatusov RL, Koonin EV, Lipman DJ: A genomic perspective on protein families. Science 1997, 278:631-637.

17. Gattiker A, Michoud K, Rivoire C, Auchincloss AH, Coudert E, Lima T, Kersey P, Pagni M, Sigrist CJ, Lachaize C, Veuthey AL, Gasteiger E, Bairoch A: Automated annotation of microbial proteomes in SWISS-PROT. Comput Biol Chem 2003, 27:49-58.

18. Riley M, Serres MH: Interim report on genomics of Escherichia coli. Annu Rev Microbiol 2000, 54:34I-4II.

19. Ren SX, Fu G, Jiang XG, Zeng R, Miao YG, Xu H, Zhang YX, Xiong $H$, Lu G, Lu LF, jiang HQ, Jia J, Tu YF, Jiang JX, Gu WY, Zhang YQ, Cai Z, Sheng HH, Yin HF, Zhang Y, Zhu GF, Wan M, Huang HL, Qian Z, Wang SY, Ma W, Yao ZJ, Shen Y, Qiang BQ, Xia QC, Guo XK, Danchin A, Saint Girons I, Somerville RL, Wen YM, Shi MH, Chen Z, Xu JG, Zhao GP: Unique physiological and pathogenic features of Leptospira interrogans revealed by whole-genome sequencing. Nature 2003, 422:888-893.

20. Nascimento AL, Ko Al, Martins EA, Monteiro-Vitorello CB, Ho PL, Haake DA, Verjovski-Almeida S, Hartskeerl RA, Marques MV, Oliveira MC, Menck CF, Leite LC, Carrer H, Coutinho LL, Degrave WM, Dellagostin OA, El-Dorry H, Ferro ES, Ferro MI, Furlan LR, Gamberini M, Giglioti EA, Goes-Neto A, Goldman GH, Goldman MH, Harakava R, Jeronimo SM, Junqueira-de-Azevedo IL, Kimura ET, Kuramae EE, Lemos EG, Lemos MV, Marino CL, Nunes LR, de Oliveira RC, Pereira GG, Reis MS, Schriefer A, Siqueira W], Sommer P, Tsai SM, Simpson AJ, Ferro JA, Camargo LE, Kitajima JP, Setubal JC, Van Sluys MA: Comparative genomics of two Leptospira interrogans serovars reveals novel insights into physiology and pathogenesis. J Bacteriol 2004, 186:2164-2I72.

21. Nascimento AL, Verjovski-Almeida S, Van Sluys MA, MonteiroVitorello CB, Camargo LE, Digiampietri LA, Harstkeerl RA, Ho PL, Marques MV, Oliveira MC, Setubal JC, Haake DA, Martins EA: 
Genome features of Leptospira interrogans serovar Copenhageni. Braz J Med Biol Res 2004, 37:459-477.

22. Soma A, Ikeuchi $Y$, Kanemasa S, Kobayashi K, Ogasawara N, Ote T, Kato J, Watanabe K, Sekine Y, Suzuki T: An RNA-modifying enzyme that governs both the codon and amino acid specificities of isoleucine tRNA. Mol Cell 2003, I 2:689-698.

23. Yan $Q$, Guan $M X$ : Identification and characterization of mouse TRMU gene encoding the mitochondrial 5-methylaminomethyl-2-thiouridylate-methyltransferase. Biochim Biophys Acta 2004, 1676:119-126.

24. Sekowska $A$, Danchin $A$ : The methionine salvage pathway in Bacillus subtilis. BMC Microbiol 2002, 2:8.

25. Reader JS, Metzgar D, Schimmel P, de Crecy-Lagard V: Identification of four genes necessary for biosynthesis of the modified nucleoside queuosine. J Biol Chem 2004, 279:6280-6285.

Publish with Bio Med Central and every scientist can read your work free of charge

"BioMed Central will be the most significant development for disseminating the results of biomedical research in our lifetime. "

Sir Paul Nurse, Cancer Research UK

Your research papers will be:

- available free of charge to the entire biomedical community

- peer reviewed and published immediately upon acceptance

- cited in PubMed and archived on PubMed Central

- yours - you keep the copyright

Submit your manuscript here:

http://www.biomedcentral.com/info/publishing_adv.asp
BioMedcentral 